\title{
Broad protection against influenza infection by vectored immunoprophylaxis in mice
}

\begin{abstract}
Neutralizing antibodies that target epitopes conserved among many strains of influenza virus have been recently isolated from humans. Here we demonstrate that adeno-associated viruses (AAV) encoding two such broadly neutralizing antibodies are protective against diverse influenza strains. Serum from mice that received a single intramuscular AAV injection efficiently neutralized all $\mathrm{H} 1, \mathrm{H} 2$ and $\mathrm{H} 5$ influenza strains tested. After infection with diverse strains of H1N1 influenza, treated mice showed minimal weight loss and lung inflammation. Protection lasted for at least 11 months after AAV injection. Notably, even immunodeficient and older mice were protected by this method, suggesting that expression of a monoclonal antibody alone is sufficient to protect mice from illness. If translated to humans, this prophylactic approach may be uniquely capable of protecting immunocompromised or elderly patient populations not reliably protected by existing vaccines.
\end{abstract}

Alejandro B Balazs ${ }^{1}$, Jesse D Bloom ${ }^{2}$, Christin M Hong ${ }^{1}$, Dinesh S Rao ${ }^{3}$ \& David Baltimore ${ }^{1}$

Influenza causes seasonal epidemics that lead on average to $>20,000$ deaths each year in the United States ${ }^{1}$ and many more throughout the world ${ }^{2}$. Neither immunological memory from prior infections nor current vaccines reliably provide lasting protection against seasonal epidemics, primarily because influenza undergoes rapid antigenic evolution that renders immune memory obsolete ${ }^{3}$. The seasonal influenza vaccine is repeatedly reformulated in an attempt to keep pace with viral evolution, but it provides little protection against highly diverged influenza strains that periodically emerge from animal reservoirs to initiate human influenza pandemics ${ }^{4}$. Zoonotic transmissions of influenza to humans have caused four global pandemics in the last 100 years, the most deadly of which is estimated to have killed 50 million people worldwide 5 . Another shortcoming of existing influenza vaccines is their markedly reduced efficacy in the elderly ${ }^{6}$, a group that suffers nearly $90 \%$ of all deaths from seasonal influenza ${ }^{1,7}$.

These facts underscore the need for new influenza vaccination paradigms capable of eliciting effective immunity to many influenza strains and in all recipients, regardless of their age or immune status. Raising hope that such vaccines might be possible, recent studies isolated human antibodies that neutralize diverse influenza strains by binding to conserved epitopes on the stalk of the hemagglutinin (HA) protein $^{8-10}$ (Fig. 1a). In general, whereas the HA head is highly variable, the stalk is relatively well conserved among distinct strains of influenza. Vaccination strategies using new prime-boost regimes ${ }^{11}$ or engineered 'headless' HA proteins ${ }^{12}$ have elicited modest levels of broadly reactive and neutralizing antibodies in mice, ferrets and monkeys. In addition, novel adjuvants have been developed that can enhance the immunogenicity of influenza vaccination ${ }^{13}$. However, to be effective, these approaches still require a functional immune response to the vaccine immunogen. As such, it is unclear whether these strategies will be sufficient to protect vulnerable patient populations (e.g., the elderly and immunocompromised individuals) who do not respond effectively to immunization.

Here, we describe an alternative means of generating broadly protective humoral immunity against influenza in both healthy and immunocompromised animals. This approach, termed vectored immunoprophylaxis (VIP), uses adeno-associated virus (AAV) to direct muscle tissue to express previously isolated and characterized broadly neutralizing monoclonal antibodies. VIP with broadly neutralizing antibodies or antibody-like immunoadhesins facilitates highly effective protection against HIV infection in humanized mice ${ }^{14}$ and simian immunodeficiency virus infection in macaques ${ }^{15}$. Here, we use VIP to generate broadly protective humoral immunity against diverse influenza strains (Fig. 1b) in healthy and immunocompromised mice.

To test the ability of VIP to protect mice from influenza infection, we inserted the variable regions from the heavy and light chains of the F10 (ref. 8) and CR6261 (refs. 9,10) broadly neutralizing influenza antibodies (Fig. 1a) into our previously described AAV expression system $^{14}$. To improve antibody serum levels in mice, we optimized the light chain variable region sequence (Supplementary Results). As a control, we used AAV vectors expressing the HIV-neutralizing b12 antibody as described previously ${ }^{14}$.

A single intramuscular injection of $1 \times 10^{11}$ genome copies (GC) of AAV into BALB/c mice resulted in expression of detectable levels of human IgG in the serum within 1 week of injection (Fig. 1c). As observed previously in immunocompetent animals ${ }^{14}$, antibody concentrations transiently declined before increasing over the following $6-8$ weeks, reaching a plateau of $50-200 \mu \mathrm{g} / \mathrm{ml}$ that was maintained for the duration of the 64-week study. The CR6261 antibody, and to a lesser degree F10 antibody, exhibited substantial mouse-to-mouse variation in expression levels for up to 5 weeks after vector administration, but expression was more uniform among mice at later time points (Supplementary Fig. 1a).

To determine the breadth of neutralizing activity of sera from VIPtreated animals, we performed in vitro neutralization assays using

${ }^{1}$ Division of Biology, California Institute of Technology, Pasadena, California, USA. ${ }^{2}$ Basic Sciences Division, Fred Hutchinson Cancer Research Center, Seattle, Washington, USA. ${ }^{3}$ Department of Pathology and Laboratory Medicine, David Geffen School of Medicine, University of California at Los Angeles, Los Angeles, California, USA. Correspondence should be addressed to D.B. (baltimo@caltech.edu). 
Figure 1 VIP results in expression of broadly neutralizing influenza antibodies in mouse serum. (a) Crystal structure of the HA trimer from the PR/34 strain of influenza (PDB code $1 R \cup 7)$. Residues are colored according to their variability in the five influenza strains used in this study, with the most variable residues shown in red and invariant residues shown in green. Variability is calculated as the site entropy. The binding of the $V_{H} / V_{L}$ domains of the F10 (red) and CR6261 (blue) antibodies is modeled in the context of the HA trimer (PDB code 1RU7) based on alignment with PDB structures of antibodies bound to HA monomer (PDB codes $3 F K U$ and $3 G B N$, respectively). These antibodies bind to the conserved stalk rather than the variable head of the HA.

(b) Matrix of pairwise identities of the $\mathrm{HA}$ protein sequences used in this study calculated as the fraction of amino acids that were identical between two sequences and colored according to the degree of identity (green is most similar and red is least similar). PR/34: A/PR/8/1934 (H1N1);

a

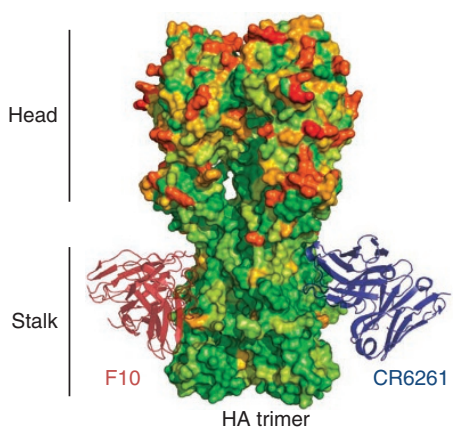

b

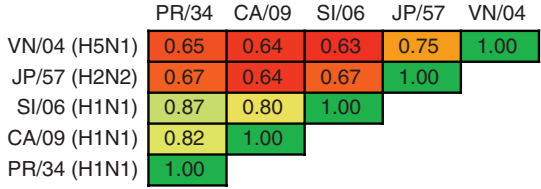

C

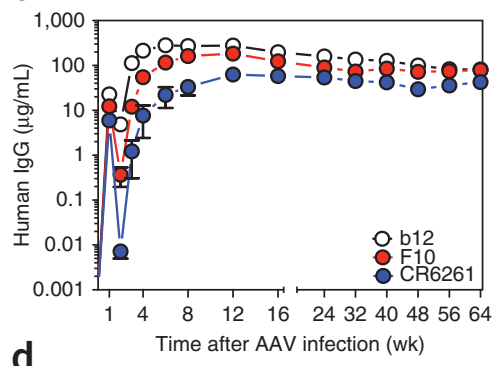

d

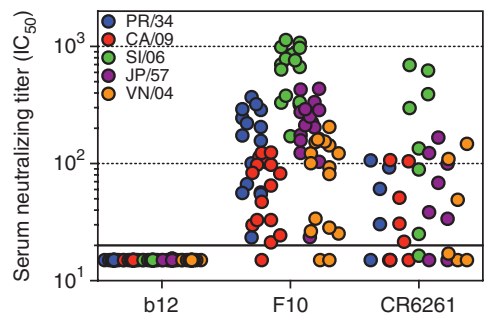

CA/09: A/California/7/2009 (H1N1); SI/06: A/Solomon Islands/3/2006 (H1N1); JP/57: A/Japan/305/1957 (H2N2); VN/04: A/Vietnam/1203/2004 (H5N1). (c) Human IgG in serum of BALB/c mice (as quantified by ELISA) at indicated times after intramuscular injection of $1 \times 10^{11}$ GC of optimized AAV encoding b12, F10 or CR6261. The plot shows mean and standard error of six recipient mice per antibody. (d) Neutralizing activity of sera taken from mice 5 weeks after they were injected with AAV encoding b12, F10 or CR6261 (just before infection with influenza virus) as measured against five strains of influenza (PR/34, CA/09, SI/06, JP/57 and VN/04) using a GFP reporter assay (Supplementary Fig. 2). Values are calculated as the fold dilution of serum that resulted in $50 \%$ neutralization of influenza infection. Solid line represents the lowest dilution tested (20-fold) and values below this line are extrapolated from the curve fit or are plotted at a value of 15 to represent no detectable neutralization activity. Each point represents an individual mouse serum sample tested against one strain of influenza. Each plot contains data generated from one individual experiment, and each experiment was performed once with the indicated number of mice.

GFP-reporter influenza virions ${ }^{16}$, each expressing one of five HA proteins; these HA proteins differ in sequence by up to $37 \%$, and originate from three different HA subtypes (H1, H2 and H5; Fig. 1b and Supplementary Fig. 2). Sera from mice expressing the b12 control antibody demonstrated no appreciable neutralization of any of the five influenza strains tested (Fig. 1d). In contrast, sera from animals that received VIP expressing either F10 or CR6261 neutralized multiple, often all five, influenza strains (Fig. 1d); the potency of neutralization activity directly correlated with the serum concentration of antibody detected by enzyme-linked immunosorbent assay (ELISA; Supplementary Fig. 1b,c).

To determine whether VIP protected mice from influenza infection, we injected AAV encoding b12, F10 or CR6261 into the gastrocnemius muscle of BALB/c mice; 5 weeks later and immediately before challenge with influenza viruses we had titered previously (Supplementary Fig. 3), we detected $\sim 100-200 \mu \mathrm{g} / \mathrm{ml}$ of b12 and F10 antibodies and a broad range of CR6261 antibody concentrations $(0.1-100 \mu \mathrm{g} / \mathrm{ml})$ in the serum of the injected mice (Fig. 2a). Following intranasal administration of 10,000 plaque-forming units (PFU) of influenza with the HA and neuraminidase (NA) from the 2009 swine-origin pandemic H1N1 strain A/California/07/2009 (CA/09), we observed dramatic weight loss in animals expressing the control b12 antibody, but no appreciable weight loss in mice expressing the F10 antibody (Fig. 2b). Interestingly, mice expressing CR6261 demonstrated a range of weight loss that was inversely proportional to the serum human IgG concentration, suggesting that a minimum serum concentration of $\sim 7.5 \mu \mathrm{g} / \mathrm{ml}$ of this antibody was required to prevent illness from CA/09 infection (Fig. 2c and Supplementary Fig. 1d). To examine the ability of VIP to protect against infection with other influenza strains, we infected recipients of b12 or F10 VIP with influenza carrying the HA and NA from the human seasonal
H1N1 strain A/Solomon Islands/3/2006 (SI/06) (Fig. 2d). Again, mice expressing b12 exhibited weight loss over a period of 2 weeks. In contrast, mice expressing F10 showed no signs of illness after SI/06 challenge. To determine the ability of VIP to protect animals from a lethal influenza challenge, we intranasally administered 1,000 PFU of the mouse-lethal H1N1 strain A/Puerto Rico/8/1934 (PR/34). As expected, mice expressing b12 control rapidly lost weight to an extent that exceeded the mandated endpoint in our study within 4 days (Fig. 2e). In contrast, mice expressing F10 showed no significant signs of illness or weight loss. Notably, when these mice were re-infected with heterologous influenza strains, we again observed protection in all cases (Supplementary Fig. 4). Together, these findings demonstrate that VIP-treated animals were protected against at least three diverse influenza strains.

Next, we determined the impact of VIP-mediated antibody expression on the endogenous humoral immune response to influenza infection. Sera from mice that expressed the b12 control antibody and that had been infected with CA/09 or SI/06 strains demonstrated strong neutralizing activity against the infection strain, but no detectable neutralizing activity against heterologous strains (Fig. 2f,g). This outcome was analogous to recent observations in humans previously vaccinated with seasonal H1N1 strains (such as SI/06), who developed antibodies against similar seasonal H1N1 strains, but produced little neutralizing antibody against highly diverged pandemic $\mathrm{H} 1 \mathrm{~N} 1$ strains (such as CA/09) ${ }^{17}$. In contrast, serum from mice expressing AAVencoded F10 or CR6261 demonstrated broad neutralizing activity against all influenza strains tested (Fig. 2f,g). To further characterize the nature of the endogenous influenza-neutralizing antibodies in these mice, we analyzed sera by hemagglutinin inhibition assays (HAI), which detect antibodies specific for the variable head (rather than the conserved stalk) of the HA protein. In sera of b12-expressing 

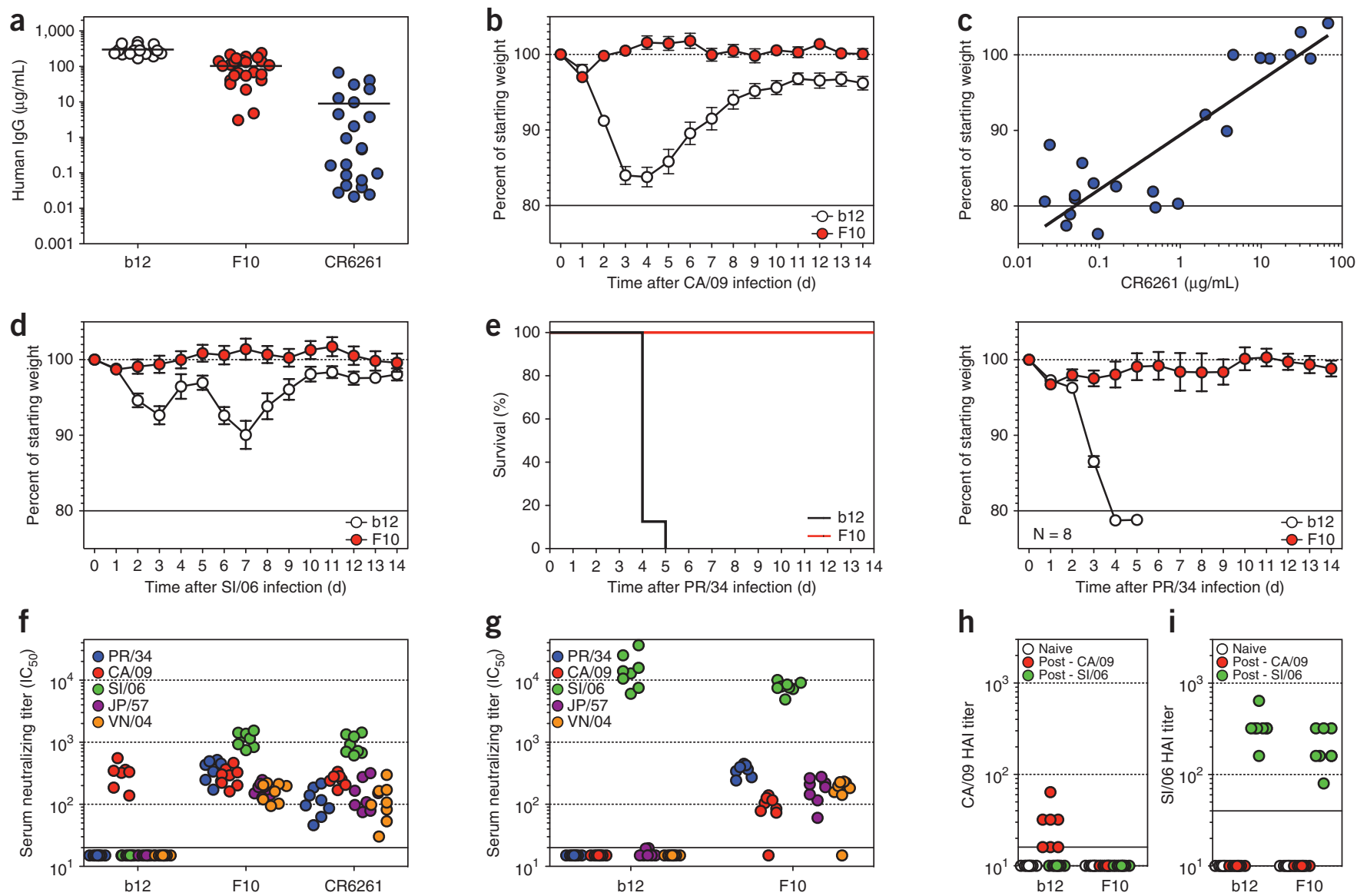

Figure 2 VIP protects mice against influenza infection. (a) Total human IgG in serum was measured by ELISA in samples taken 5 weeks after intramuscular injection of AAV encoding b12, F10 or CR6261 (2 d before influenza infection) into BALB/c mice. Each point represents an individual mouse. (b,c) BALB/c mice that received VIP 5 weeks prior were infected intranasally with $1 \times 10^{4}$ PFU of CA/09 influenza. Weight loss was monitored for the indicated time period in recipients of AAV encoding control (b12) or F10. The plot shows mean and standard error of eight recipient mice per antibody (b). Correlation between weight loss of individual mice $4 \mathrm{~d}$ post-infection with CA/09 and serum concentration of CR6261 (c). (d) BALB/c mice that received VIP 5 weeks prior were infected intranasally with $5 \times 10^{4} \mathrm{PFU}$ of SI/06 influenza. Weight loss was monitored for the indicated time period in recipients of AAV encoding control (b12) or F10. The plot shows mean and standard error of eight recipient mice per antibody. (e) BALB/c mice that received VIP 5 weeks prior were infected intranasally with 1,000 PFU of PR/34 influenza. Survival (left) and weight loss (right) were monitored for the indicated time period in recipients of AAV encoding control (b12) or F10. The plots shows mean and standard error of eight recipient mice per antibody. (f,g) Serum was taken from BALB/c mice 8 weeks after they were injected with AAV encoding b12, F10 or CR6261, and 3 weeks after they were infected with CA/09 (f) or SI/06 (g) influenza. In vitro neutralization of five strains of influenza (PR/34, CA/09, SI/06, JP/57 and VN/04) by serum was detected by GFP-reporter assays. Values are calculated as the fold dilution that resulted in $50 \%$ neutralization of influenza infection. Solid line represents the lowest dilution tested (20-fold dilution), and values below this line are extrapolated from the curve fit or are plotted at a value of 15 to represent no detectable neutralization activity. (h,i) Serum was taken from BALB/c mice 5 weeks after they were injected with AAV encoding b12 or F10 and either just before (naive) or 3 weeks after (post-) infection with CA/09 or SI/06. HAI performed with CA/09 virus (h) or SI/06 virus (i). HAI titers are expressed as the highest dilution of serum that allowed complete hemagglutination. Solid line represents the lowest dilution tested (16-fold or 40-fold dilution for $\mathrm{CA} / 09$ or $\mathrm{SI} / 06$, respectively), and values below this line are plotted along the axis to represent no detectable HAI activity. Each plot contains data generated from one individual experiment, and each experiment was performed once with the indicated number of mice.

mice, we detected endogenous HAI activity specific for the influenza strain that had infected the mice but not for heterologous strains. In contrast, in F10-expressing mice that had been infected with CA/09, we did not detect endogenous CA/09 HAI activity above the detection threshold (Fig. 2h). However, in F10-expressing mice that had been infected with SI/06, we detected endogenous SI/06-specific HAI activity (Fig. 2i). This difference may result from the higher dose of SI/06 inoculum administered, which may have provided more HA antigen. These results suggest that under certain conditions, protection by broadly neutralizing antibodies can still allow the formation of endogenous humoral immunity specific to an influenza strain.

Given the robust protection of young, immunocompetent mice treated with VIP, we sought to determine whether such an approach would be effective in older or immunocompromised animals. For these experiments, we employed nonobese diabetic/severe combined immunodeficient (NOD.SCID). $\mathrm{Il}_{2 \mathrm{rg}^{-/-}}$(NSG) mice that completely lack adaptive immune cells and also exhibit markedly impaired innate immune responses ${ }^{18}$. To account for variation in mouse weight, we administered AAV encoding luciferase or antibody at a dose of $5 \times 10^{12}$ GC per kg to NSG animals; we intramuscularly injected both relatively young (14-19 weeks old) and old (46-55 weeks old) animals, and quantified expression of proteins encoded by AAV for 4 weeks using bioluminescence imaging or ELISA (Fig. 3a,b). Young and old mice showed similar abundance of AAV-encoded proteins at all time points, suggesting that age did not affect the capacity for muscle-based gene expression from AAV. To determine whether VIP 

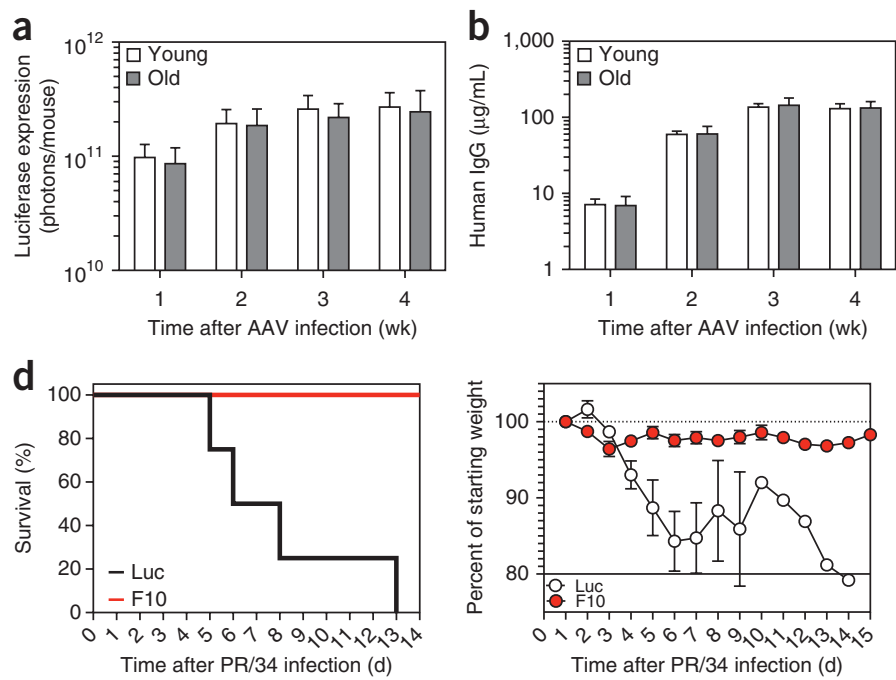

Figure 3 VIP protects both young and old immunodeficient mice from influenza infection. (a) Young (3 months) or old (12 months) NSG mice were injected intramuscularly with $1 \times 10^{11} \mathrm{GC}$ of AAV encoding luciferase. At indicated time points, luciferase was measured in vivo by bioluminescence imaging. The plot shows mean and s.d. of eight young and eight old recipient mice. (b) Young or old NSG mice were injected intramuscularly with $1 \times 10^{11} \mathrm{GC}$ of AAV encoding F10. At indicated time points, serum human IgG was measured by ELISA. The plot shows mean and s.d. of eight young and eight old recipient mice. (c,d) Young (c) and old (d) NSG mice were injected intramuscularly with $1 \times 10^{11} \mathrm{GC}$ of AAV encoding F10 or luciferase (Luc). Four weeks later mice were infected intranasally with 1,000 PFU of PR/34 influenza. Survival (left) and weight loss (right) were measured $(n=4-8)$. Error bars, mean and s.e.m. (e) Hematoxylin and eosin staining of representative lung sections from young NSG mice that received VIP encoding F10 or Luc, taken $5 \mathrm{~d}$ after infection with 1,000 PFU of PR/34 influenza. Scale bar, $100 \mu \mathrm{M}$. (f-h) BALB/c mice were injected with AAV encoding b12 or F10. Eleven months later, total human IgG in serum was quantified by ELISA (1 d before influenza infection) (f). Each point represents an individual mouse. (g,h) Eleven months after injection of AAV, mice were infected with 1,000 PFU of PR/34 influenza $(n=14-20)$. Weight loss was monitored $(g)$. Error bars, mean and s.e.m. Immediately following infection and every $2 \mathrm{~d}$ thereafter, three mice were euthanized in each group and influenza RNA in the lungs was measured by qPCR ( $h$ ). Samples were normalized to the endogenously expressed mouse ribosomal protein L32 gene and plotted relative to the signal of the lowest sample. Each plot contains data generated from one individual experiment, and each experiment was performed once with the indicated number of mice.

was sufficient to protect these immunocompromised animals from challenge with influenza, we administered 1,000 PFU of the lethal $\mathrm{PR} / 34$ strain to young and old mice 4 weeks after AAV administration (Fig. 3c,d). In both cases, we observed weight loss that reached the study endpoint for euthanasia in all control NSG mice that received VIP encoding luciferase. In contrast, both young and old NSG mice that received VIP encoding F10 were completely protected from influenza-induced weight loss, indicating that the VIP-expressed F10 antibody alone was sufficient to protect mice in the absence of an endogenous humoral or T-cell immune response. To further characterize the extent to which VIP-driven F10 expression was capable of preventing illness in young NSG mice, we contemporaneously euthanized an equal number of protected F10-expressing animals together with luciferase-expressing animals as they reached the mandated study endpoint and scored the level of inflammation in histological samples of lung tissue. Lungs taken from VIP-luciferase mice $5 \mathrm{~d}$ after influenza infection demonstrated substantial luminal infiltration of the bronchioles (Fig. 3e, left and Supplementary Figs. 5 and 6). In contrast, lungs taken from VIP-F10 mice showed very low levels of inflammation and clear bronchioles, consistent with a substantially lower level of pathology in these mice (Fig. 3e, right and Supplementary Figs. 5 and 6). We scored lung inflammation and measured viral RNA in lung samples taken at various time points after influenza infection, and found that both young and old mice
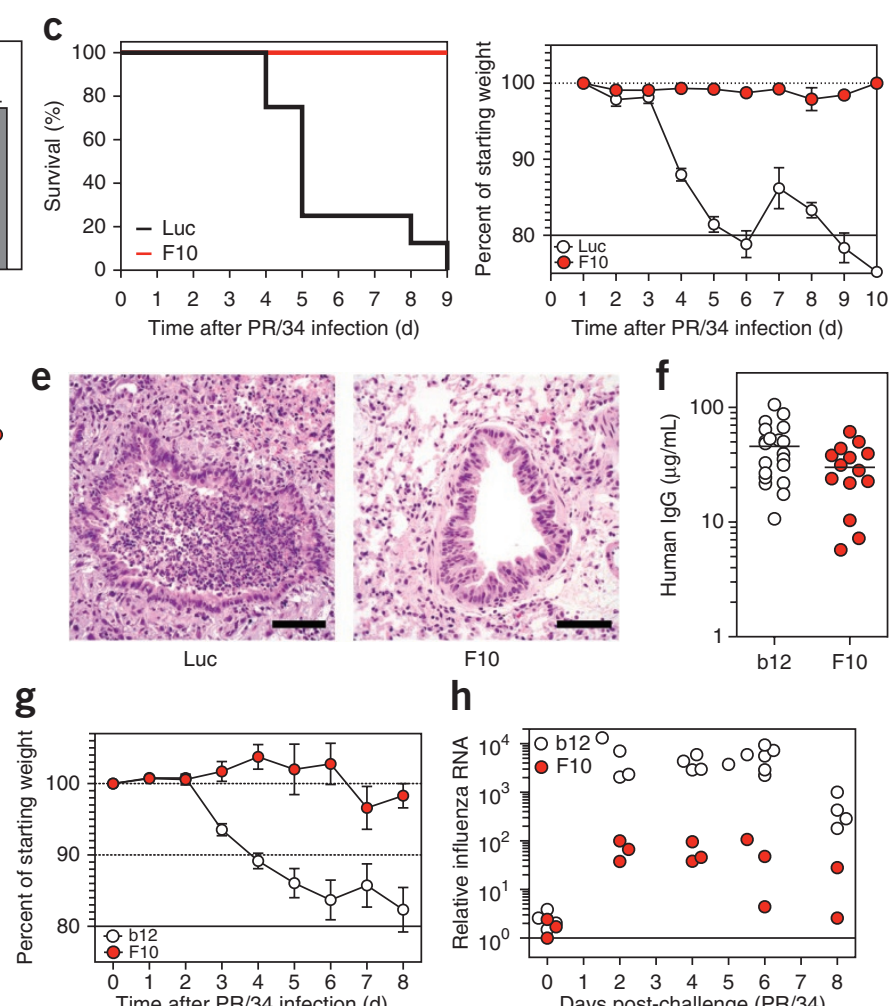

h

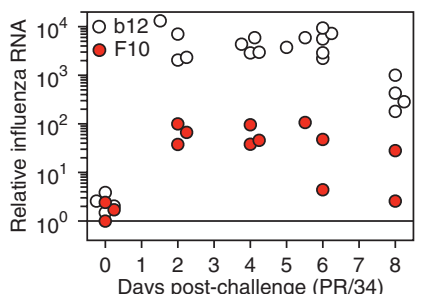

exhibited the most severe inflammation and highest viral load at earlier time points (Supplementary Fig. 7a). However, compared to VIP-luciferase mice, VIP-F10 mice exhibited less inflammation and viral load at all time points analyzed. To directly quantify the ability of VIP to control viral replication in NSG mice, we examined the amount of virus present in the lungs by extracting total RNA from lung tissue harvested at the time of death and measured viral RNA by qPCR. Lungs from mice expressing luciferase exhibited high viral load throughout the course of the experiment (Supplementary Fig. 7b). In contrast, F10-expressing animals analyzed at early time points contained moderate levels of viral RNA that declined substantially over time, as a result of dramatically reduced viral replication in the presence of F10 antibody despite the lack of endogenous adaptive immunity.

To determine the longevity of VIP-mediated protection, we administered AAV encoding b12 or F10 to BALB/c mice and quantified serum human IgG after 11 months. We detected levels of b12 and F10 antibody $>10 \mu \mathrm{g} / \mathrm{ml}$ in the sera of most of these animals (Fig. 3f). We subsequently infected these mice intranasally with 1,000 PFU of PR/34 influenza. Mice expressing the b12 but not the F10 antibody exhibited extensive weight loss, suggesting that recipients of F10-encoding VIP were protected from illness (Fig. 3g). To quantify the growth of influenza in the lungs of these mice, we euthanized animals every $3 \mathrm{~d}$ after influenza infection, isolated lung tissue and measured the 
abundance of influenza RNA by qPCR. We detected lower amounts of influenza RNA in the lungs of F10- compared to b12-expressing animals, suggesting that the presence of antibody inhibited the growth of the virus but did not completely prevent influenza infection and viral replication (Fig. $3 \mathbf{h}$ ).

To examine the effectiveness of VIP in a large animal model of influenza, we administered b12 or F10-expressing AAV by intramuscular injection to ferrets. Although we detected production of human antibody in these animals, the titers achieved were $\sim 100$-fold lower than those observed in mice, and did not result in protection against influenza challenge (data not shown). This expression difference may be the result of the substantially reduced half-life of human IgG1 in ferrets as compared to mice (W. Strohl, personal communication), or the substantial immunogenicity we observed in ferrets against the human antibody proteins (data not shown), which was not seen in mice (Supplementary Fig. 8), and which would not be predicted to affect expression in humans.

Taken together, our results demonstrate that a single intramuscular injection of AAV encoding an influenza broadly neutralizing antibody is capable of protecting mice against diverse influenza strains. Such broad efficacy could be especially important during pandemics, when pre-existing immunity and conventional vaccines are unable to protect against newly emerging HA subtypes. Recent work demonstrated that only a handful of mutations are necessary to enable the highly pathogenic $\mathrm{H} 5 \mathrm{~N} 1$ avian strain to transmit by airborne routes in ferrets ${ }^{19}$. If such a strain were to begin transmitting between humans, preparation of suitable quantities of a vaccine (at least using currently approved methods) would require many months ${ }^{20}$. Even if the HA subtype of a pandemic strain was known before a pandemic outbreak, efforts to stockpile a traditional vaccine against this subtype would be of uncertain value because ongoing antigenic evolution may render such vaccines ineffective ${ }^{21}$. In contrast, VIP can administer antibodies that neutralize diverse influenza strains of multiple HA subtypes, including $\mathrm{H} 5$. In addition, because AAV is a remarkably stable vector that can be lyophilized ${ }^{22}$, VIP may be effectively stockpiled before the start of a pandemic. Furthermore, increasingly potent and broad influenza-neutralizing antibodies continue to be discovered ${ }^{23-26}$, and their incorporation into VIP could extend the effectiveness of this approach to group 2 influenza strains (such as H7) and perhaps to all known influenza A and B strains.

The ability of VIP to protect animals lacking a properly functioning immune system is particularly important when one considers that the vast majority of deaths from seasonal influenza occur in the elderly ${ }^{1}$. Because current strategies to elicit protection against influenza still require an effective immune response, elderly individuals ${ }^{7}$, and individuals with weakened immune systems ${ }^{27}$, are less likely to be protected from infection. As VIP results in production of broadly neutralizing antibodies without involving the immune system, it could be employed to protect individuals who do not respond to traditional vaccination.

Although the concentration of antibody that can be produced by VIP in humans has yet to be determined, recent studies have demonstrated expression of therapeutic levels of factor IX using AAV8-based vectors in human patients ${ }^{28}$. If similar clinical success is achieved for VIP, it may provide a plausible approach to protect patients, especially those who are most vulnerable, against both epidemic and pandemic influenza.

\section{METHODS}

Methods and any associated references are available in the online version of the paper.
Note: Supplementary information is available in the online version of the paper.

\section{ACKNOWLEDGMENTS}

The authors wish to thank D. Majumdar, A. Sigal and J. Zhao for their helpful comments, and other members of the Baltimore Lab for their assistance in carrying out this work. This project was supported by a contract from the National Institute of Allergy and Infectious Disease (NIAID) and by the Joint Center for Translational Medicine as well as a Caltech Translational Innovation Partnership Award.

A.B.B. is supported by the amfAR postdoctoral research fellowship no. 107756-47RFVA. D.S.R. is supported by career development award 1K08CA133521 from the National Institutes of Health. The bidirectional reverse-genetics plasmids ${ }^{29}$ from the PR/34 strain of influenza were a kind gift from R. Webby of St. Jude Children's Research Hospital. The pHW2000 plasmid was a kind gift from Y. Kawaoka of the University of Wisconsin.

\section{AUTHOR CONTRIBUTIONS}

A.B.B. conceived the study, designed and performed experiments, and wrote the manuscript. J.D.B. conceived the study and designed experiments, C.M.H. performed experiments, D.S.R. performed histological analysis, and D.B. conceived the study and wrote the manuscript.

\section{COMPETING FINANCIAL INTERESTS}

The authors declare no competing financial interests.

Reprints and permissions information is available online at http://www.nature.com/ reprints/index.html.

1. Centers for Disease Control and Prevention (CDC). Estimates of deaths associated with seasonal influenza-United States, 1976-2007. MMWR Morb. Mortal. Wkly. Rep. 59, 1057-1062 (2010).

2. Nair, H. et al. Global burden of respiratory infections due to seasonal influenza in young children: a systematic review and meta-analysis. Lancet 378, 1917-1930 (2011).

3. Smith, D.J. et al. Mapping the antigenic and genetic evolution of influenza virus Science 305, 371-376 (2004).

4. Morens, D.M. \& Taubenberger, J.K. Historical thoughts on influenza viral ecosystems, or behold a pale horse, dead dogs, failing fowl, and sick swine. Influenza Other Respir. Viruses 4, 327-337 (2010).

5. Johnson, N.P.A.S. \& Mueller, J. Updating the accounts: global mortality of the 1918-1920 'Spanish' influenza pandemic. Bull. Hist. Med. 76, 105-115 (2002).

6. Aspinall, R., Del Giudice, G., Effros, R.B., Grubeck-Loebenstein, B. \& Sambhara, S. Challenges for vaccination in the elderly. Immun. Ageing 4, 9 (2007).

7. Goodwin, K., Viboud, C. \& Simonsen, L. Antibody response to influenza vaccination in the elderly: a quantitative review. Vaccine 24, 1159-1169 (2006).

8. Sui, J. et al. Structural and functional bases for broad-spectrum neutralization of avian and human influenza A viruses. Nat. Struct. Mol. Biol. 16, 265-273 (2009).

9. Ekiert, D.C. et al. Antibody recognition of a highly conserved influenza virus epitope Science 324, 246-251 (2009).

10. Throsby, M. et al. Heterosubtypic neutralizing monoclonal antibodies cross-protective against $\mathrm{H} 5 \mathrm{~N} 1$ and $\mathrm{H} 1 \mathrm{~N} 1$ recovered from human IgM+ memory B cells. PLOS ONE 3, e3942 (2008).

11. Wei, C.-J. Induction of broadly neutralizing H1N1 influenza antibodies by vaccination. Science 329, 1060-1064 (2010).

12. Steel, J. et al. Influenza virus vaccine based on the conserved hemagglutinin stalk domain. mBio 1, e00018-10 (2010).

13. Clark, T.W. et al. Trial of 2009 influenza A (H1N1) monovalent MF59-adjuvanted vaccine. N. Engl. J. Med. 361, 2424-2435 (2009).

14. Balazs, A.B. et al. Antibody-based protection against HIV infection by vectored immunoprophylaxis. Nature 481, 81-84 (2011).

15. Johnson, P.R. et al. Vector-mediated gene transfer engenders long-lived neutralizing activity and protection against SIV infection in monkeys. Nat. Med. 15, 901-906 (2009).

16. Bloom, J.D., Gong, L.I. \& Baltimore, D. Permissive secondary mutations enable the evolution of influenza oseltamivir resistance. Science 328, 1272-1275 (2010).

17. Hancock, K. et al. Cross-reactive antibody responses to the 2009 pandemic H1N1 influenza virus. N. Engl. J. Med. 361, 1945-1952 (2009).

18. Shultz, L.D. et al. Human lymphoid and myeloid cell development in NOD/LtSz-scid IL2R gamma null mice engrafted with mobilized human hemopoietic stem cells. J. Immunol. 174, 6477-6489 (2005).

19. Enserink, M. Infectious diseases. Controversial studies give a deadly flu virus wings. Science 334, 1192-1193 (2011).

20. Collin, N. \& de Radiguès, X. World Health Organization H1N1 Vaccine Task Force Vaccine production capacity for seasonal and pandemic (H1N1) 2009 influenza. Vaccine 27, 5184-5186 (2009). 


\section{LETTERS}

21. Li, Y. et al. Continued evolution of $\mathrm{H} 5 \mathrm{~N} 1$ influenza viruses in wild birds, domestic poultry, and humans in China from 2004 to 2009. J. Virol. 84, 8389-8397 (2010)

22. Croyle, M.A., Cheng, X. \& Wilson, J.M. Development of formulations that enhance physical stability of viral vectors for gene therapy. Gene Ther. 8, 1281-1290 (2001).

23. Corti, D. et al. A neutralizing antibody selected from plasma cells that binds to group 1 and group 2 influenza A hemagglutinins. Science 333, 850-856 (2011).

24. Marsh, G.A., Hatami, R. \& Palese, P. Specific residues of the influenza A virus hemagglutinin viral RNA are important for efficient packaging into budding virions. J. Virol. 81, 9727-9736 (2007).
25. Krause, J.C. et al. A broadly neutralizing human monoclonal antibody that recognizes a conserved, novel epitope on the globular head of the influenza H1N1 virus hemagglutinin. J. Virol. 85, 10905-10908 (2011).

26. Dreyfus, C. et al. Highly conserved protective epitopes on influenza B viruses. Science 337, 1343-1348 (2012).

27. Anonymous. Recommendations of the Advisory Committee on Immunization Practices (ACIP): use of vaccines and immune globulins for persons with altered immunocompetence. MMWR Recomm. Rep. 42, 1-18 (1993).

28. Nathwani, A.C. et al. Adenovirus-associated virus vector-mediated gene transfer in hemophilia B. N. Engl. J. Med. 365, 2357-2365 (2011). 


\section{ONLINE METHODS}

Influenza reverse-genetics plasmids. The bidirectional reverse-genetics plasmids ${ }^{29}$ from the PR/34 strain of influenza were a kind gift from Richard Webby of St. Jude Children's Research Hospital. The plasmids encoding the HA and NA genes from the CA/09, SI/06 and JP/57 influenza strains were generated by cloning the influenza genes by reverse-transcription from viral RNA obtained from the BEI Resources reagents program into the pHW2000 plasmid, a kind gift from Yoshihiro Kawaoka of the University of Wisconsin. The gene for the VN/04 HA was synthesized commercially, and then cloned into this plasmid.

Influenza virus production and quantification. All influenza viruses used for the mouse infections in this study derived their six internal genes (PB2, PB1, PA, NP, M and NS) from the A/Puerto Rico/8/1934 (H1N1) strain. The HA and NA genes were derived from the following strains and given the following name abbreviations:

- PR/34: the HA and NA derived from A/Puerto Rico/8/1934 (H1N1), a widely used laboratory-adapted strain.

- CA/09: the HA and NA derived from A/California/07/2009 (H1N1), a strain isolated early during the emergence in humans of the 2009 swine-origin H1N1 pandemic.

- SI/06: the HA and NA derived from A/Solomon Islands/3/2006 (H1N1), a human seasonal H1N1 vaccine strain.

The viruses were generated using the 8-plasmid bidirectional reverse-genetics system $^{29}$. Briefly, 293T and MDCK cells were maintained in DMEM (Mediatech) supplemented with 10\% FBS (Omega Scientific), 100 IU/ml penicillin (Mediatech), $100 \mu \mathrm{g} / \mathrm{ml}$ streptomycin (Mediatech) and 1\% L-glutamine (Mediatech). Six-well tissue culture dishes (Corning) containing co-cultures of $293 \mathrm{~T}$ and MDCK cells were co-transfected with $250 \mathrm{ng}$ of each of the eight plasmids. At $14 \mathrm{~h}$ post-transfection, the media was aspirated, the cells were washed once with PBS and influenza growth medium plus $3 \mu \mathrm{g} / \mathrm{ml}$ of TPCKtreated trypsin (Sigma-Aldrich) was added to the cells. Influenza growth medium consists of Opti-MEM I (Invitrogen) with $0.01 \%$ FBS, $0.3 \%$ bovine serum albumin (Invitrogen), $100 \mathrm{IU} / \mathrm{ml}$ penicillin, $100 \mu \mathrm{g} / \mathrm{ml}$ streptomycin and $100 \mu \mathrm{g} / \mathrm{ml}$ calcium chloride. After $72 \mathrm{~h}$, the supernatant was collected and passaged to $15-\mathrm{cm}$ dishes (Corning) containing nearly confluent MDCK cells in influenza growth medium plus $3 \mu \mathrm{g} / \mathrm{ml}$ trypsin. After $72 \mathrm{~h}$, the viral supernatant was harvested and centrifuged at 2,000 g for $5 \mathrm{~min}$. The viral supernatant was removed and aliquoted, and the aliquots were frozen at $-80{ }^{\circ} \mathrm{C}$.

Plaque assays. The influenza viruses were quantified by plaque assays on MDCK cells using an Avicel microcrystalline cellulose overlay ${ }^{30}$. Briefly, MDCK cells were seeded into 6-well tissue culture dishes. When the cells were $95 \%$ confluent, the media was removed and serial tenfold dilutions of viral inoculum were added to a $1-\mathrm{ml}$ final volume of influenza growth medium. After $40 \mathrm{~min}$, the inoculum was removed by aspiration and replaced by $4 \mathrm{ml}$ of influenza growth media with $2.4 \%$ Avicel microcrystalline cellulose and $3 \mu \mathrm{g} / \mathrm{ml}$ of TPCK-treated trypsin. The plates were grown undisturbed for $3 \mathrm{~d}$ at $37^{\circ} \mathrm{C}$. The overlay was then removed by aspiration, the cell layer was washed twice with PBS and the cells were stained with $0.1 \%$ crystal violet in $20 \%$ ethanol for $15 \mathrm{~min}$. This staining solution was then removed by aspiration, the cells were washed again with PBS and the plaques were counted visually to determine the viral titer in terms of plaque-forming units (PFU).

Mouse strains. Immunocompetent $\mathrm{BALB} / \mathrm{cJ}(\mathrm{BALB} / \mathrm{c})$ and immunodeficient NOD.SCID.Il2 $\mathrm{rg}^{-1-}$ (NSG) mice of $\sim 4-5$ weeks of age were obtained from the Jackson Laboratory (JAX). For experiments involving aged mice, these animals were bred and housed under barrier conditions for the indicated period of time before influenza challenge. All experiments were conducted in accordance with the policies of the California Institute of Technology institutional animal care and use committee (IACUC).

Cloning of influenza-neutralizing antibodies into AAV vector. Sequences corresponding to the heavy and light chain variable regions were synthesized (Integrated DNA Technologies) and used as templates for overlapping
PCR reactions designed to fuse the variable regions into the IgG1 constant region framework present in the VIP expression vector as previously described $^{14}$. Subsequent optimization of the light chains required to achieve efficient expression in vivo are described in the Supplementary Results and Supplementary Figures 9-12.

AAV production. AAV production and intramuscular injection were done as previously described ${ }^{14}$. Briefly, $1.2 \times 10^{8} 293$ T cells were transfected with $80 \mu \mathrm{g}$ of the vector encoding the antibody of interest, pHELP (Applied Viromics), and pAAV 2/8 SEED (University of Pennsylvania Vector Core) at a ratio of 0.25:1:2. Supernatant was collected 5 times over the course of $120 \mathrm{~h}$. Virus was purified by PEG precipitation and cesium chloride fractionation before being diafiltrated, concentrated and buffer exchanged through $100 \mathrm{k}$ MWCO centrifuge filters (Millipore) into buffer consisting of $100 \mathrm{mM}$ sodium citrate and $10 \mathrm{mM}$ Tris $\mathrm{pH} 8$ before aliquoting and storage at $-80^{\circ} \mathrm{C}$. To quantify aliquots, virus was thawed, treated with DNase and titered by qPCR as previously described ${ }^{14}$. Briefly, virus titer was determined by quantitative PCR using a standard curve generated from previously titered, purified, AAV2/8 encoding 4E10 antibody. Infectivity of virus aliquots was confirmed in vitro by transducing $293 \mathrm{~T}$ cells and quantifying antibody concentration in the cell supernatant by ELISA.

AAV administration to mice. Before intramuscular injection, virus was thawed and diluted to the indicated dose with buffer ( $100 \mathrm{mM}$ sodium citrate, $10 \mathrm{mM}$ Tris $\mathrm{pH} 8$ ) in a $40 \mu \mathrm{L}$ volume. Mice were anesthetized by isoflorane inhalation, and virus was administered as a single injection of $40 \mu$ into the gastrocnemius muscle.

In recipients of luciferase-encoding $\mathrm{AAV}$, bioluminescent imaging was done using an IVIS 200 instrument essentially as previously described ${ }^{14}$ Bioluminescent images were taken 10 min after intraperitoneal injection of $1.5 \mathrm{mg}$ D-luciferin (Gold Biotechnology). In recipients of antibody-encoding $\mathrm{AAV}$, the concentration of human IgG in mouse serum was determined by performing ELISAs using a standard curve generated from purified Human IgG/Kappa (Bethyl).

Infection of mice with influenza. Influenza viruses were thawed and diluted in PBS to deliver the indicated dose in a $20 \mu \mathrm{l}$ volume. Prior to inoculation, mice were weighed and anesthetized by intraperitoneal injection of $200 \mu \mathrm{l}$ of a cocktail containing $2 \mathrm{mg}$ of ketamine and $0.2 \mathrm{mg}$ xylazine diluted in PBS. Mice were infected with influenza by intranasal inoculation with $20 \mu \mathrm{l}$ of diluted virus, $10 \mu \mathrm{l}$ per nostril. Infected mice were weighed at the same time each day.

GFP influenza virus production and quantification. PB1flank-GFP influenza viruses were generated by a previously described system in which GFP is packaged in the PB1 segment ${ }^{16}$. PB1flank-GFP viruses were grown and assayed in 293T-CMV-PB1 and MDCK-SIAT1-CMV-PB1 cells that supplied the missing $\mathrm{PB} 1$ protein in trans, as described ${ }^{16}$. PB1flank-GFP viruses were generated using the 8-plasmid bidirectional reverse-genetics system ${ }^{29}$, but with the standard PB1 plasmid replaced by $\mathrm{pHH}-\mathrm{PB} 1$ flank-eGFP ${ }^{16}$. For these viruses, the other five internal genes (PB2, PA, NP, M and NS) were derived from the PR/34 strain as for the viruses used in the mouse infections. In addition to viruses with the HA and NA from PR/34, CA/09 and SI/06, we also used two additional viruses in these assays:

- JP/57: the HA and NA derived from A/Japan/305/1957 (H2N2), an early strain from the Asian flu pandemic.

- VN/04: the HA from A/Vietnam/1203/2004 (H5N1), a highly pathogenic avian influenza strain. The NA for this virus was derived from the laboratoryadapted A/WSN/1933 (H1N1) strain. The polybasic cleavage site was removed from the HA.

PB1flank-GFP viruses were quantified by flow cytometry. MDCK-SIAT1CMV-PB1 cells were seeded in 12-well dishes (Corning) at $10^{5}$ cells per well in $1 \mathrm{ml}$ of influenza growth medium; $8 \mathrm{~h}$ after seeding, viruses were diluted $1: 10,1: 100$ and 1:1000 in media. Wells were infected with $50 \mu \mathrm{l}$ of each of these dilutions. Cells were harvested $16.5 \mathrm{~h}$ post-infection by incubation with 
$250 \mu \mathrm{l}$ of trypsin-EDTA (Invitrogen) for $5 \mathrm{~min}$, removal of trypsin by aspiration and resuspension in $250 \mu \mathrm{l}$ of PBS supplemented with $2 \%$ FBS and $2 \mu \mathrm{g} / \mathrm{ml}$ propidium iodide (Invitrogen). Samples were analyzed on a FACSCalibur flow cytometer (Becton-Dickinson), and samples with a percentage of GFP-positive cells between $0.3 \%$ and $3 \%$ were used to quantify viral titer. Titer was calculated from the percentage of GFP-positive cells, the dilution factor and the total count of $10^{5}$ cells per well.

Neutralization assays. Neutralization assays were performed using PB1flankGFP influenza viruses and MDCK-SIAT1-CMV-PB1 cells. We added $40 \mu \mathrm{l}$ of influenza growth medium to all wells of a flat-bottom 96-well tissue culture dish (Corning), except for row A, which received $57 \mu \mathrm{l}$ of media. Mouse sera samples were serially diluted by adding $3 \mu \mathrm{l}$ of serum to the $57 \mu \mathrm{l}$ of influenza growth medium in row $A$, then performing $1: 3$ serial dilutions down to row $G$, resulting in an initial dilution of 1:20 and final dilution of $1: 4.374 \times 10^{4}$. We added $2 \times 10^{4}$ infectious particles of PB1flank-GFP virus (as determined by flow cytometry titering) to samples in a $20 \mu \mathrm{l}$ volume of influenza growth medium. The mixtures of diluted serum and virus were incubated for $1 \mathrm{~h}$ in a $5 \% \mathrm{CO}_{2}$ incubator at $37^{\circ} \mathrm{C}$. After incubation, $2 \times 10^{4} \mathrm{MDCK}-\mathrm{SIAT} 1-\mathrm{CMV}-\mathrm{PB} 1$ cells in a $20 \mu \mathrm{l}$ volume of influenza growth medium were added to all wells for an MOI of 1 . A cell-only control, which received naive BALB/c mouse serum and no virus, and a virus-only control, which received naive $B A L B / c$ mouse serum, were included for each virus strain. Plates were incubated in a 5\% $\mathrm{CO}_{2}$ incubator at $37^{\circ} \mathrm{C}$ for $18 \mathrm{~h}$. Post-incubation, $40 \mu \mathrm{l}$ of $1.5 \%$ Triton X-100 (Sigma-Aldrich) in PBS was added to each well to give a final concentration of $0.5 \%$ Triton X-100, and plates were incubated at room temperature for $5 \mathrm{~min}$. We transferred $100 \mu \mathrm{l}$ of each sample into opaque 96-well plates (Corning) for reading. GFP fluorescence was quantified using a Safire2 plate reader (Tecan) configured to read from the top with an excitation of $485 \mathrm{~nm}$, emission of $515 \mathrm{~nm}, 12-\mathrm{nm}$ slit widths for both excitation and emission, gain set to "optimal," an integration time of $500 \mu$ s and 5 reads per well. Baseline fluorescence from the cell-only control was subtracted from all readings. Samples were normalized to the virus-only control.

HAI assays. The HAI assay was done as described in the WHO Animal Influenza Manual (WHO/CDS/CSR/NCS/2002.5). Chicken red blood cells (Lampire) and 96-well V-bottom plates (Costar) were used for all assays. Hemagglutination units (HAU) of stock SI/06 and CA/09 were determined by diluting virus 1:50 in $1 \times \mathrm{PBS}$, $\mathrm{pH} 7.2-7.4$, then serially twofold in a final volume of $25 \mu \mathrm{l} /$ well. Influenza virus was diluted to $4 \mathrm{HAU} / 25 \mu \mathrm{l}$ immediately before use in the HAI assay. RDE (receptor-destroying enzyme) (Seiken) was reconstituted in physiological saline $(0.85 \% \mathrm{NaCl})$ according to manufacturer instructions. Mouse sera was diluted 1:4 in reconstituted RDE and left overnight at $37^{\circ} \mathrm{C}$; the following day, RDE was inactivated by incubation at $56^{\circ} \mathrm{C}$ for $30 \mathrm{~min}$. Sera were diluted either 1:40 (SI/06) or 1:16 (CA/09) in physiological saline and serially twofold diluted in a volume of $25 \mu \mathrm{l} /$ well.
We added $4 \mathrm{HAU}$ of virus to each well in a volume of $25 \mu \mathrm{l}$. Controls were run on each plate to confirm HAU and RBC activity. After a 15-min incubation at room temperature, $50 \mu \mathrm{l}$ of $0.5 \%$ chicken red blood cells were added to each well. Plates were incubated for $30 \mathrm{~min}$ at room temperature. HAI titer was defined as the reciprocal of the greatest dilution that completely inhibited hemagglutination of RBCs.

Histology. At the conclusion of the in vivo challenge experiments, lungs were removed from mice and half of this tissue was immersed in $10 \%$ neutral buffered formalin for $24 \mathrm{~h}$. Following fixation, tissues were removed from formalin and placed in $70 \%$ ethanol until standard paraffin embedding and processing. Four-micron-thick sections were then cut, and stained with hematoxylin and eosin staining (H\&E). The slides were reviewed by a pathologist (D.S.R.) on an Olympus BX51 light microscope, and images were obtained using a SPOT Insight Digital Camera (Diagnostic Instruments). Inflammation was scored as follows: $0=$ no to minimal inflammation; $1=$ occasional infiltrates in bronchioles (less than $10 \%$ of bronchioles); 2 = easily identified infiltrates in bronchioles (10-50\% of bronchioles); 3 = easily identified infiltrates in bronchioles with parenchymal infiltrates and/or early patchy fibrosis; $4=>50 \%$ of bronchioles with infiltrates, or 10-50\% bronchiole involvement with extensive necrotic epithelium in bronchioles, angionecrosis or extensive fibrosis. Scoring was done in a blinded fashion and an ordinal scale was assumed for any statistical tests.

Relative viral quantification by qPCR. Lung tissue was homogenized in $100 \mu \mathrm{l}$ PBS. We used $25 \mu \mathrm{l}$ of homogenate for RNA extraction via TRIzol Reagent (Invitrogen). Purified RNA was resuspended in nuclease-free water, and RNA concentration was normalized to $150 \mathrm{ng} / \mu \mathrm{l}$. Real-time RT-qPCR was performed using qScript One-Step SYBR Green qRT-PCR Kit, Rox (Quanta Biosciences) with primers designed against $\mathrm{PR} / 34 \mathrm{M}$ as described ${ }^{24}$ as PR8 M and an endogenous control consisting of mouse ribosomal protein L32 with the following sequences:

Forward PR8-M: CAAGCAGCAGAGGCCATGGA, Reverse PR8-M: GAC CAGCACTGGAGCTAGGA, Forward-L32: AAGCGAAACTGGCGGAAAC Reverse-L32: TAACCGATGTTGGGCATCAG. Samples were treated with DNase using Turbo DNase kit (Invitrogen) and run in triplicate on an ABI 7300 Real-Time PCR System (Life Technologies) with the following program: $50^{\circ} \mathrm{C}$ for $10 \mathrm{~min}, 95^{\circ} \mathrm{C}$ for $5 \mathrm{~min}, 40$ cycles of $95^{\circ} \mathrm{C}$ for $15 \mathrm{~s}$ and $55^{\circ} \mathrm{C}$ for $30 \mathrm{~s}$, followed by a melt curve analysis. Each sample was individually normalized by L32 signal to account for variation in input RNA.

29. Hoffmann, E., Neumann, G., Kawaoka, Y., Hobom, G. \& Webster, R.G.A. DNA transfection system for generation of influenza A virus from eight plasmids. Proc. Natl. Acad. Sci. USA 97, 6108-6113 (2000).

30. Matrosovich, M., Matrosovich, T., Garten, W. \& Klenk, H.-D. New low-viscosity overlay medium for viral plaque assays. Virol. J. 3, 63 (2006). 